\title{
Zeitenwende im Büro: Angestelltenarbeit im Sog der Globalisierung
}

Andreas Boes

Tobias Kämpf

\begin{abstract}
Wer sich über die Globalisierung und deren Folgen für Arbeit und Beschäftigung Gedanken machte, hatte bisher zumeist Industriearbeiter vor Augen. Mit der Diskussion um Offshoring hat sich dieses Szenario verändert - die Globalisierung erreicht nun immer öfter auch die Büros der Angestellten und Hochqualifizierten. Unsere Untersuchungen in den Feldern Informationstechnologie (IT), Shared Services sowie Forschung und Entwicklung (F\&E) zeigen, dass damit für diese Beschäftigtengruppe neue Zeiten anbrechen. Dies bleibt auch für das Bewusstsein der Beschäftigten nicht folgenlos: Während die Identifikation mit den Unternehmen abnimmt, zeigen sich gleichzeitig Tendenzen in Richtung eines neuen Arbeitnehmerbewusstseins.
\end{abstract}

\section{Einleitung: Eine neue Phase der Globalisierung}

Der folgende Artikel fragt nach den Folgen einer neuen Phase der Globalisierung (Boes et al. 2008) für die Arbeitsbedingungen und die Arbeitsbeziehungen in qualifizierten und hochqualifizierten Angestelltenbereichen. Diesen Bereichen ist gemein, dass sie bislang kaum im Fokus der Globalisierung standen - sie galten vielmehr sogar als „geschützt“. Verlagert und internationalisiert wurden die Fabriken der Industriearbeiter, nicht aber die Büros, die Forschungseinrichtungen und die Dienstleistungsjobs der Angestellten.

Hier deutet sich heute jedoch eine bemerkenswerte Wende an. Unter dem Label Offshoring geraten nun auch jene Arbeitsbereiche unter den Druck der Globalisierung, die bislang als weitgehend verlagerungsresistent galten (einen Überblick über die Diskussion bieten Boes 2004, 2005 und Kämpf 2008; siehe auch Ahlers et al. 2007). Insbesondere Länder wie Indien und China sowie Mittelosteuropa gelten nun als attraktive und kostengünstige Alternativen zu den traditionellen High-Tech-Standorten der westlichen Welt.

Im Zentrum dieser Entwicklung stehen drei große Bereiche der Angestelltenarbeit:

- die Internationalisierung von SoftwareEntwicklung und IT-Dienstleistungen,
- der Aufbau sogenannter Shared Services Center in Niedriglohnländern, in denen Unternehmen Verwaltungstätigkeiten wie zum Beispiel Buchhaltung, Reisekostenabrechnung u.ä. konzentrieren,

- die Internationalisierung der Forschungs- und Entwicklungsabteilungen großer Unternehmen, die nun auch in Niedriglohnländern eigene Entwicklungsstandorte etablieren.

Die Basis dieser neuen Phase der Globalisierung bildet ein grundlegender Produktivkraftsprung (ausführlich dazu Boes/ Kämpf 2010). Globale Informations- und Kommunikationstechnologie-Netze und das Internet werden dabei zur Infrastruktur eines neuen, global verfügbaren „Informationsraums“ (Baukrowitz/Boes 1996). Dieser ist nicht nur Infrastruktur zum bloßen Transport digitalisierter Information, sondern erweist sich als neuer „sozialer Handlungsraum“ (Boes 1996). Über große Entfernungen können Menschen darin sozial interagieren. Mit Blick auf Arbeit bedeutet dies, dass Menschen auch im Arbeitsprozess von unterschiedlichen Orten aus in Echtzeit kooperieren können, da ihr Arbeitsgegenstand (zum Beispiel eine Software-Applikation) im Informationsraum zugänglich ist. Auch wenn dabei die konkreten Standorte keineswegs an Bedeutung verlieren und Unternehmen nicht „footloose“ werden, wird der globale Informationsraum so für diejenigen Formen der Kopfarbeit, deren wesentliche Arbeitsgegenstände und -mittel digitalisierbar sind, zu einem weltweiten „Raum der Produktion“ (Boes 2004, 2005).

Unsere empirischen Untersuchungen zeigen, dass in allen drei oben genannten Feldern der Angestelltenarbeit die Globalisierung auch in der Praxis tatsächlich zu greifen beginnt und für die Beschäftigten in neuer Qualität zu einer neuen Kontextbedingung ihrer Arbeit wird - auch wenn dabei die jeweils eigene Entwicklungslogik der Felder und organisatorische, soziale und kulturelle Schranken nicht übersehen werden dürfen (ausführlich dazu Boes/ Kämpf 2010). Am weitesten fortgeschritten sind die Internationalisierungsprozesse im Bereich Software-Entwicklung und ITDienstleistungen (zur Hardware-Industrie siehe Lüthje 2006; Hürtgen et al. 2008).

\footnotetext{
Andreas Boes, PD Dr. phil., ist Vorstandsmitglied des Instituts für Sozialwissenschaftliche Forschung e.V. (ISF) München und Privatdozent an der TU Darmstadt. Arbeitsschwerpunkte: Informatisierung der Gesellschaft, Zukunft der Arbeit, Globalisierung, Entwicklung der Arbeitsbeziehungen, Gesundheit und Prävention. e-mail: andreas.boes@isf-muenchen.de Tobias Kämpf, Dr. phil., ist wissenschaftlicher Mitarbeiter am ISF München. Arbeitsschwerpunkte: Globalisierung und Offshoring, Hochqualifizierte und Mitbestimmung, Angestelltensoziologie, Gesundheit und Prävention. e-mail: tobias.kaempf@isf-muenchen.de
} 
Hier sind global integrierte Produktionsund Geschäftsmodelle mittlerweile zum globalen Benchmark geworden. In Bereichen wie der F\&E erhält die Globalisierung ebenfalls eine neue Bedeutung. Hier wird intensiv nach neuen Formen international verteilter Kopfarbeit gesucht (vgl. dazu auch Ernst 2008), ohne dabei primär auf Arbeitsplatzverlagerungen abzuzielen. Während es hier vorrangig um die Etablierung globaler Entwicklungsnetzwerke geht, wird im Bereich der Shared Services der Fokus auf Kostensenkung und Verlagerung gerichtet.

Dieser Globalisierungsschub im Angestelltenbereich geht in den drei Feldern mit einer starken Betonung der Standardisierung und einer verstärkten Prozessorientierung einher (ausführlich dazu Boes 2004, 2005; Boes/Kämpf 2010). Oft bilden dabei Standardisierungen von Produkten und Prozessen die Voraussetzung, um die Arbeit international verteilt erbringen $\mathrm{zu}$ können. Umgekehrt gilt jedoch auch, dass sich der steigende globale Wettbewerb als Treiber der Standardisierung von Arbeit erweist. Globalisierung und Standardisierung verstärken sich so wechselseitig - und bilden als zwei Säulen eines ,informatisierten Produktionsmodus" (Boes/ Kämpf 2008, 2010) die Grundlage für eine Verschiebung zentraler Koordinaten qualifizierter und hochqualifizierter Angestelltenarbeit.

Es gilt nun zu fragen, wie sich in der Folge die Arbeitsbedingungen und Arbeitsbeziehungen in den neu von der Globalisierung erfassten Bereichen verändern. Um auch die Konsequenzen für die Arbeitspolitik bestimmen zu können, wollen wir mit Blick auf unsere empirischen Ergebnisse insbesondere diskutieren, wie sich diese Veränderungen in den Köpfen der betroffenen qualifizierten und hochqualifizierten Angestelltengruppen niederschlagen.

\section{Stand der Forschung und offene Fragen}

Mit Blick auf den hier zur Debatte stehenden Zusammenhang von Globalisierung und Angestellten kreist die arbeits- und industriesoziologische Diskussion derzeit vor allem um die Frage nach einer neuen „transnationalen Managerklasse“. Aus- gehend von prominenten Zeitdiagnosen, wie zum Beispiel Manuel Castells' Aussage „elites are cosmopolitan, people are local“ (Castells 1996), wird diskutiert, ob und in welcher Form das Management tatsächlich Transnationalisierungs- bzw. Kosmopolitisierungsprozessen unterworfen ist (vgl. z.B. Beck 2008; Dahrendorf 2009; Hartmann 2009). Unabhängig davon, wie man zu den Ergebnissen dieser Diskussion steht - empirische Untersuchungen legen eine gewisse Skepsis nahe (vgl. z. B. Hartmann 2009) -, fällt auf, dass sich die Debatte auf die oberen Ränge des Managements konzentriert. Auch wenn einzelne Studien durchaus die Widersprüche und Belastungen für die global tätigen Grenzgänger im Management thematisieren (Minssen 2009), hat die Frage nach den Folgen globalisierter Wertschöpfungsprozesse für qualifizierte und hochqualifizierte Angestellte in dieser Debatte bisher wenig Gewicht. Abgesehen von einzelnen Ausnahmen wie z.B. die Forschungsarbeiten von Flecker/ Huws (2004) und Huws/Dahlmann (2009) wurde so die Bedeutung der Globalisierung für die Entwicklung der Angestelltenarbeit unterhalb des oberen Managements empirisch kaum beforscht.

Blickt man auf die lebhaft geführte Angestelltendiskussion seit den 1970er Jahren zurück, standen zunächst weniger die Führungskräfte im Zentrum, sondern die Frage, wie und ob sich Angestellte und Arbeiter unterscheiden und ob eine Angleichung ihrer Lagen absehbar sei (sehr lesenswert dazu Kadritzke 1982). Ausgehend von den Überlegungen von Harry Braverman (1977) wurde nach den Möglichkeiten der Rationalisierung von Angestelltentätigkeiten gefragt. Braverman nahm an, dass Verwaltungsarbeitsplätze im Büro sowie Ingenieurstätigkeiten zunehmend Gegenstand einer Taylorisierung würden, die, da diese für ihn gewissermaßen als Wesen kapitalistischer Verwertung galt, schließlich zu einer umfassenden Proletarisierung führen würde. In der Praxis erwies sich eine große Zahl der adressierten Tätigkeiten als wenig zugänglich für tayloristische Rationalisierungsstrategien (vgl. z. B. Littek/Heisig 1995) - und auch die sozialwissenschaftliche Debatte fokussierte nun mehr das „Rationalisierungsdilemma der Angestelltenarbeit“ (Berger/ Offe 1981), aber auch das Entstehen alternativer Rationalisierungsformen (Baethge/ Oberbeck 1986). Die in diesem Kontext angestellten Überlegungen inspirierten auch eine intensive gewerkschaftliche Diskussion zur „Angestelltenfrage“ (vgl. dazu z.B. Bleicher 1985; Urban 1989).

In den 1990er Jahren verlor dieser zentrale Diskussionsstrang zur Rationalisierung von Angestelltenarbeit und deren Folgen für das Bewusstsein von Angestellten wie auch für die gewerkschaftliche Arbeitspolitik an Dynamik. Im Anschluss an diegrundlegendeStudie „Das Führungskräfte-Dilemma“ (Baethge et al. 1995) konzentrierte sich die Angestelltendiskussion nunmehr stattdessen auf leitende Angestellte und Führungskräfte (so auch Kotthoff 1997; Faust et al. 2000; aktuell z. B. Kotthoff/Wagner 2008; Hoose et al. 2009). Die Etablierung neuer Managementkonzepte und der Lean Production brachte, so der Tenor der Debatte, nun auch untere und mittlere Managementgruppen unter Druck. Empirisch eindrucksvoll wurde gezeigt, dass tief greifende Veränderungstendenzen in den Unternehmen wie z. B. Dezentralisierung, eine verstärkte Orientierung am Shareholder Value oder Restrukturierungs- und Kostensenkungsprogramme - die Arbeitssituation und Rolle der Führungskräfte beeinträchtigten und im Sinne von „Widerspruchserfahrungen" (Boes/Trinks 2006) auch zu ersten Rissen in den Interessen- und Arbeitsorientierungen der leitenden Angestellten führten - die sich resümierend eher als Knirschen im Gebälk erwiesen, jedoch die soziale Bindung an die Unternehmen nicht grundlegend erodieren ließen. Zugespitzt formuliert, verhielten sich die Führungskräfte trotz aller Widerspruchserfahrungen zu Gewerkschaften und Betriebsräten weiterhin zurückhaltend bis ablehnend.

Mit dem Hype um die New Economy erfuhr die Angestelltendiskussion eine radikale Wende. Es ging nun zunehmend weniger um die Frage, ob sich Angestellte und Hochqualifizierte Gewerkschaften und Betriebsräten zuwenden könnten. Stattdessen firmierten die zumeist hochqualifizierten Angestelltengruppen der New Economy nun als Protagonisten eines neuen Modells von Arbeit und Arbeitsbeziehungen. In diesem Zukunftsentwurf erschienen Gewerkschaften als Auslaufmodell, selbstbewusste Beschäftigte verträten ihre Interessen vielmehr individuell. Aus arbeitspolitischer Perspektive bestimmten deshalb der "Arbeitskraftunternehmer“ (Voß/Pongratz 1998) und die These einer Durchsetzung individueller gegenüber kollektiven Formen des Interessenhan- 
delns die Richtung der Debatte (Heidenreich/Töpsch 1998).

Mit dem Zusammenbruch der New Economy erodierte dieses Zukunftsszenario. Auch die Protagonisten der New Economy verloren nun ihren Sonderstatus. Die Diskussion zu diesem Veränderungsprozess wird in Deutschland vor allem mit Blick auf die IT-Beschäftigten geführt. Zum einen gibt es dabei Stimmen, die betonen, dass insbesondere im kleinbetrieblich strukturierten Segment der Start-up-Firmen der dot.com-Generation trotz einer gewissen Ernüchterung die individuelle Selbstvertretung der Königsweg der meisten Beschäftigten bleibe (vgl. z. B. Lange et al. 2005; Abel et al. 2005). Zum anderen gibt es jedoch auch Studien, die argumentieren, dass in Folge der Krise und damit verbundener Konflikte und Verwerfungen auch kollektive Formen des Interessenhandelns an Legitimität gewinnen (vgl. z. B. Boes/Trinks 2006; Martens 2006; Mehlis 2008; Kämpf 2008; Martens/ Dechmann 2010). Mit Blick auf eine neue Qualität von Widerspruchserfahrungen und einen tief greifenden Wandel des Bewusstseins der zumeist hochqualifizierten Beschäftigen sprechen deshalb Boes und Trinks (2006) zugespitzt von einer „Zeitenwende " für Hochqualifizierte im Gefolge der Krise der New Economy.

Die hier zur Diskussion gestellten Ergebnisse knüpfen an diese Befunde an. Heute zeigt sich, dass sich mit der Durchsetzung globaler Produktionsstrukturen in qualifizierten und insbesondere hochqualifizierten Bereichen der Angestelltenarbeit Widerspruchserfahrungen weiter vertiefen und verstetigen. Auch wenn diese Felder einer Taylorisierung zumeist weiterhin unzugänglich bleiben, führt das Zusammenwirken von Globalisierung und Standardisierung zu einer grundlegenden Veränderung ihrer Arbeitssituation. Zum Kern der "Zeitenwende im Büro" wird dabei ein veränderter Umgang der Unternehmen mit den Angestellten, die in der Folge ihre besondere, mitunter privilegierte Position im Unternehmen verlieren. Die Beschäftigten erleben diesen Veränderungsprozess im Sinne neuer Lohnarbeitserfahrungen, in deren Folge sich selbst in hochqualifizierten Angestelltenbereichen neue Arbeitnehmeridentitäten herausbilden. Diese Entwicklung muss nicht zwangsläufig zu einer Aufwertung von Betriebsräten und Gewerkschaften führen. Sie kann auch die Gewerkschafts- und mit- bestimmungskritischen Haltungen in diesen Feldern weiter verstärken - gleichzeitig sind damit jedoch auch neue Chancen und Potenziale für Gewerkschaften verbunden, in Angestelltenbereichen in neuer Qualität Fuß zu fassen.

Die Grundlage unserer Überlegungen bilden mehrere empirische Forschungsprojekte zur Entwicklung von Angestelltenarbeit. Im Zentrum stehen dabei acht Fallstudien in den Untersuchungsfeldern Software-Entwicklung und IT-Dienstleistungen (vier Fälle), Verwaltung (zwei Fälle) sowie F\&E (zwei Fälle), die wir im Rahmen des Projekts „Offshoring und eine neue Phase der Internationalisierung von Arbeit" erhoben haben. ${ }^{1}$ Dabei wurden insgesamt 102 Interviews geführt. Neben 37 Expertengesprächen mit Managementvertretern, internationalen Projektleitern, Betriebsräten und Gewerkschaftsvertretern konnten 66 Intensivinterviews mit Beschäftigten durchgeführt werden. Sowohl qualifizierte Angestellte mit einer dualen Ausbildung (20) als auch hochqualifizierte Angestellte mit FH- oder Hochschulabschluss (46) wurden befragt. Von den befragten Beschäftigten sind zehn weiblich. Darüber hinaus gehen in unsere Überlegungen weitere empirische Forschungsprojekte ein, die die Entwicklung der IT-Industrie in den letzten zehn Jahren aus unterschiedlichen Perspektiven zum Gegenstand hatten. ${ }^{2}$ Dabei können wir auf insgesamt 48 Fallstudien in Deutschland, Indien, Osteuropa und den USA und mehr als 300 Interviews zurückgreifen, davon rund 150 intensive Beschäftigteninterviews. Unsere Untersuchungen konzentrieren sich vor allem auf ,ehemals fordistische Unternehmen“ sowie „Lack-TurnschuhUnternehmen“ (Boes/Baukrowitz 2002) im Bereich Software-Entwicklung und IT-Dienstleistungen. Auch wenn keine Panel-Studie im eigentlichen Sinne vorliegt, können mit Blick auf die unterschiedlichen Erhebungszeitpunkte der Projekte (19992000, 2003-2004, 2005-2009) der Wandel der Perspektive der Beschäftigten und die Entwicklung von Interessenidentitäten differenziert rekonstruiert werden.

\section{Zeitenwende im Büro}

Die Beschäftigten erleben den neuen Globalisierungsschub als einen tief greifenden
Bruch in der Entwicklung ihrer Arbeitsbedingungen und ihrer Beschäftigungssituation. Aus ihrer Perspektive brechen „neue Zeiten" an - so ein befragter Entwickler.

\subsection{DER WANDEL DER ARBEIT}

Mit der Etablierung globaler Produktionsstrukturen geht zunächst ein grundlegender Wandel der Arbeitsinhalte einher. In allen Untersuchungsfeldern konnten wir umfassende Versuche der Unternehmen rekonstruieren, die Arbeitsinhalte und -prozesse im Angestelltenbereich in neuer Qualität zu standardisieren. In der Praxis geht es nicht nur darum, die entsprechenden Wertschöpfungsprozesse zu beschleunigen, zu verschlanken und skalierbar zu machen - vielmehr geht es insbesondere darum, Kontrollierbarkeit, Planbarkeit und Wiederholbarkeit herzustellen. Die gewählten Verfahren und Instrumente in den verschiedenen Feldern - z. B. in der Software-Entwicklung und im SharedServices-Bereich - können sich mitunter erheblich unterscheiden. Was ihnen jedoch gemein ist, ist das Bestreben der Unternehmen, die Abhängigkeit vom einzelnen Beschäftigten und dessen konkreter Individualität zu reduzieren. Das Ziel ist dabei nicht, die subjektiven Potenziale der Beschäftigten im Sinne des Taylorismus aus- bzw. gleichzuschalten. Vielmehr geht es um die systematische und wiederholbare Nutzung ihrer Subjektleistung. Nicht die individuelle „Genialität“ des einzelnen Beschäftigten fungiert nunmehr als Erfolgsrezept der Unternehmen, sondern die Entwicklung robuster und stabiler Prozesse, in denen sich das Know-how und die Leistungsfähigkeit der Organisation materialisieren (Boes/Kämpf 2008). Angestrebt wird eine möglichst effiziente systemische Nutzung und Integration der geistigen Produktivkraft der Beschäftigten in die Wertschöpfung.

1 Dieses Forschungsprojekt wurde von der HansBöckler-Stiftung gefördert.

2 Zentrale Projekte in diesem Zusammenhang waren u.a. die gleichfalls von der Hans-Böckler-Stiftung geförderten Projekte "ARB-IT1“ (Boes/Baukrowitz 2002) und "ARB-IT2" (Boes/Trinks 2006) sowie das vom Bundesministerium für Bildung und Forschung (BMBF) geförderte Projekt "Export IT" (Boes et al. 2007, 2008). Gegenwärtig setzen wir unsere Forschungen zur Internationalisierung fort im Projekt „GlobePro - Global erfolgreich durch professionelle Dienstleistungsarbeit" (gefördert durch das BMBF, www.globe-pro.de). 
Die Beschäftigten stehen diesen Veränderungen oftmals sehr skeptisch gegenüber. Aus ihrer Perspektive nehmen vor allem die früher ausgeprägten Freiheitsgrade in der Arbeit ab. Auch wenn im Sinne eines Ausdifferenzierungsprozesses innovative Nischen - zum Beispiel im Bereich der Software-Architektur oder im IT-Engineering - erhalten bleiben bzw. neu entstehen, fühlt sich ein Großteil der Beschäftigten durch Standardisierungen und Prozessorientierung in seiner Kreativität und Eigenständigkeit eingeschränkt und reglementiert. Auch in Bereichen wie der Forschung und Entwicklung erleben viele Beschäftigte ihre Arbeit immer mehr durch Regeln und Prozesse bestimmt, die oftmals mit Mehrarbeit einhergehen und sie aus ihrer Perspektive nicht selten eher behindern als unterstützen. Zunehmende Formalisierungen und die verpflichtende Nutzung standardisierter Tools lösen die vormalige Praxis des „hemdsärmeligen Tüftelns" ab. Informelle Kommunikationswege verlieren angesichts zunehmend globaler Arbeitsprozesse und verteilter Teams ihre arbeitsorganisatorische Grundlage. Eine vermehrte Arbeitsteilung und Differenzierung von Rollen und Aufgaben schränkt ganzheitliche Aufgabenprofile mehr und mehr ein (vgl. dazu auch Kämpf 2008).

Die grundlegende Bedeutung dieser Veränderungen für die Beschäftigten ist kaum zu überschätzen - ist doch die besonders hohe Identifikation mit dem Arbeitsinhalt ein zentraler Bestandteil ihrer beruflichen Identität. Gleichzeitig gilt, dass in dem Maße, wie sich neue Formen der Prozessorientierung durchzusetzen beginnen, auch die Reichweite der von den Beschäftigten kontrollierten „Ungewissheitszonen“ (Crozier/Friedberg 1979) sinkt - und damit werden sie als Individuen (bzw. ihre konkrete Arbeitskraft) austauschbarer als zuvor.

\subsection{NEUE LOHNARBEITS- ERFAHRUNGEN}

Neben dem Wandel ihrer Arbeit sind Personalabbau, permanente Reorganisationsprogramme und die Verlagerung von Arbeitsplätzen der unmittelbarste Ausdruck der „neuen Zeiten“ für die Beschäftigten. Davon ist in den Fallunternehmen nun auch der Angestelltenbereich betroffen. Dies hat nicht nur bei den qualifizierten Beschäftigten, sondern insbesondere auch bei den hochqualifizierten Beschäftigten zu neuen Unsicherheiten geführt: Die Befindlichkeit vieler befragter Hochqualifizierter ist kaum mehr von einem zuversichtlichem Blick in die Zukunft geprägt, sondern vielmehr von manifesten Sorgen um die Sicherheit des eigenen Arbeitsplatzes. Auch wenn man die krisenhafte Entwicklung der Fallunternehmen in Rechnung stellt, hat uns das enorme Ausmaß der Verunsicherung der Beschäftigten überrascht. Selbst unbefristete Beschäftigungsverhältnisse vermitteln ihnen heute kaum mehr Sicherheit. Stattdessen haben viele das Gefühl, dass es auch für sie „keine Garantien" mehr gibt. Kern dieser neuen Unsicherheit ist die Erfahrung von Austauschbarkeit. Diese Unsicherheit bringt ein befragter Projektleiter sehr anschaulich zum Ausdruck:

„Und das ist ein bisschen Roulettespiel. Das ist nicht mehr so wie früher, du hast eine Karriereplanung, du planst was, und dann weißt du ganz genau: Wenn du hier drei Projekte gemacht hast, wirst du Abteilungsleiter oder Hauptabteilungsleiter - das gibt's nicht mehr, vergessen Sie's. Das kann trotzdem sein, du hast ein Superprojekt gemacht du hast 120 Mio. $€$ Umsatz gemacht und nächstes Jahr bist du nicht mehr da, Punkt. Weil du irgendwo eine Zahl in einem Planspiel bist. Du bist austauschbar, nur noch eine Zahl im Prinzip.“

Die so beschriebene Entsicherung ihrer Arbeit werten die Beschäftigten nicht als isoliertes Ereignis. Vielmehr erleben sie diese Veränderung als Teil eines schleichenden Kulturbruchs in vielen Unternehmen. Übergreifender Tenor dabei ist, „dass alles irgendwie kälter und instrumenteller wird" und dass es auf einmal nur noch um Zahlen, aber nicht mehr um die Menschen gehe. Dies gilt nicht nur für die Beschäftigten der IT-Unternehmen, wo wir einen Kulturbruch bereits im Zuge der Krise der New Economy empirisch identifizieren konnten (Boes/Trinks 2006). Interessanterweise zeigt sich, dass ein vergleichbarer kultureller Wandel von vielen Beschäftigten auch im Bereich der Verwaltung und der Forschung und Entwicklung beschrieben wird. Auch hier beklagen die Befragten, dass unter dem Druck von Sparprogrammen und Restrukturierungsmaßnahmen der Mensch immer weniger „im Mittelpunkt steht“. So argumentiert zum Beispiel ein befragter Ingenieur:
„... aber man hat irgendwie [früher] so das Soziale im Mittelpunkt gesehen. Also der Mensch ist Mittelpunkt. Das steht auch, glaub ich, immer noch in den Parolen so drin. (...) Aber es ist schon so, dass man das frühere Leitbild, dass der Mitarbeiter im Vordergrund steht, im Management ein bisschen aus dem Auge verloren wurde. Manchem aus dem Management ist das aus dem Auge gerückt. Und die haben nur noch ihren Gewinn vor Augen."

In der obigen Interviewpassage wird deutlich, dass sich auch die Beziehungen zum Management verändern. Dieses erscheint weniger als früher als ein Partner auf Augenhöhe, mit dem Entscheidungen diskursiv und auf Expertenbasis ausgehandelt werden können. Vielmehr werden heute - so die Perspektive der Beschäftigten - Entscheidungen von oben kaskadenförmig nach unten „heruntergebrochen“ und als Anweisungen bzw. Vorgaben durchgesetzt - in der Innenwelt der Unternehmen wird diese Managementpraxis nicht selten mit dem Anglizismus "to execute“ umschrieben. Entscheidungsspielräume und Freiheitsgrade in der Arbeit gehen so verloren.

Hinter der Kritik an der Ökonomisierung der Unternehmenskultur und dem neuen Führungsstil verbergen sich sehr grundlegende Verschiebungen der Anerkennungsordnungen in den Unternehmen und ein Bruch der „impliziten Verträge“ (Kotthoff 1997; Rousseau 1995; Raeder/ Grote 2001). Nicht nur als Mensch, sondern auch in ihrer Rolle als Experten und Fachkräfte fühlen sich dabei viele Beschäftigte nicht mehr anerkannt und wertgeschätzt. Dies wird von den Beschäftigten als eine umfassende Erfahrung der Entwertung geschildert - vielfach sind Frustration, Desillusionierung und Sinnverluste die Folge.

\subsection{VON DER "VERANTWORTLICHEN AUTONOMIE" ZUM "SYSTEM PERMANENTER BEWÄHRUNG ${ }^{\prime}$}

Die skizzierten Veränderungen lassen sich in ihrem Wesen als Ausdruck eines schleichenden Abschieds vom Kontrollmodus der „verantwortlichen Autonomie“ (Friedman 1977) interpretieren (kritisch dazu Kratzer/Nies 2009). Jenseits des tayloristischen „command \& control“ bildeten in diesem Kontrollmodus spezifische „implizite Verträge“ und komplementäre Anerkennungsordnungen in weiten 
Bereichen der Angestelltenarbeit die Basis eines besonderen Leistungsarrangements, das vor allem auf einer grundsätzlichen Stabilität des Arbeitsverhältnisses beruhte.

Demgegenüber haben sich heute die Vorzeichen grundlegend gedreht. Nicht mehr die vormalige „Beschaulichkeit“ so ein Befragter - der „verantwortlichen Autonomie“ prägt heute die Szenerie in den untersuchten Angestelltenbereichen, sondern die Durchsetzung eines neuen "Systems permanenter Bewährung" (Boes/ Bultemeier 2008). Dabei ist nun gerade die Sicherheit des Arbeitsplatzes keine Selbstverständlichkeit mehr. Vielmehr wird die Zugehörigkeit zum Unternehmen „optional gestellt" und an den jeweiligen Wertbeitrag des Beschäftigten zum Unternehmenserfolg gebunden (ebd.). Auch Aufstiegs- und Karrieremuster orientieren sich immer weniger am Prinzip der Seniorität, sondern an individueller Performance und Zielerreichung. Die Grundlage hierfür ist eine zunehmende informatorische Durchdringung vieler Arbeitsbereiche, die die Performance des Einzelnen und dessen Zielerreichung transparent, messbar und damit vor allem vergleichbar macht.

Damit wird die Arbeit in der Praxis für viele zur permanenten Bewährungsprobe: Täglich gilt es erneut zu zeigen, dass man es weiterhin verdient hat, dazuzugehören. Im Zeitalter der Globalisierung und weltweiter Standortkonkurrenz heißt dies zugleich, selbst als Hochqualifizierter immer öfter begründen zu müssen, warum die eigene Arbeit eigentlich nicht auch in Indien, China oder Osteuropa gemacht werden kann. In wachsenden Bereichen der Angestelltenarbeit wird damit die Erfahrung von Austauschbarkeit zu einer neuen Handlungsgrundlage - aus einer lediglich „formellen" Lohnarbeit beginnt so erst eine „echte“ Lohnarbeit zu werden (ausführlich dazu Boes/Kämpf 2008, 2010).

\section{1 \\ Wandel in den Köpfen}

Diese Veränderungen haben bei den qualifizierten und hochqualifizierten Beschäftigten nicht nur kurzfristige Irritationen ausgelöst, sondern nachhaltige Spuren in ihren Köpfen hinterlassen. Die Veränderung ihres Bewusstseins bzw. ihrer „Interessenidentitäten“ (Boes/Trinks 2006) folgt einer komplexen und mitunter wi- dersprüchlichen Entwicklungslogik. Zwei grundlegende, miteinander verbundene Entwicklungsprozesse sind dabei von entscheidender Bedeutung: auf der einen Seite ein Prozess der Erosion der vormals hegemonialen „Beitragsorientierung“ (Kotthoff 1997) und auf der anderen Seite Neuorientierungsprozesse in Richtung neuer Arbeitnehmeridentitäten (vgl. dazu auch Vester et al. 2007). Dabei entstehen neue Formen des Arbeitnehmerbewusstseins zwischen den Polen Ohnmacht und Solidarität.

Die neue Qualität der Lohnarbeitserfahrungen und der Kulturbruch in den Unternehmen veranlassen gerade die befragten Hochqualifizierten dazu, ihr Selbstverständnis als Beschäftigte grundlegend zu hinterfragen. Insbesondere die mit der Zeitenwende einhergehende einseitige Kündigung der „impliziten Verträge“ hat dazu geführt, dass das Commitment und die Identifikation der Beschäftigten mit ihren Unternehmen spürbar zurückgegangen sind. Echte Beitragsorientierte lassen sich in den von uns untersuchten Angestelltenbereichen fast nur noch im Bereich der Führungskräfte finden.

Die Beschäftigten versuchen nun in einem für sie schwierigen Lernprozess, sich vom Unternehmen und von ihrer Arbeit auch emotional stärker abzugrenzen. Dabei hat vor allem die der „Beitragsorientierung" zugrunde liegende Vorstellung einer Interesseneinheit zwischen Angestellten und Unternehmen an Überzeugungskraft eingebüßt. Die Erfahrung von Personalabbau, wiederholte Verlagerungsdrohungen und die gezielte Nutzung der Austauschbarkeit von Beschäftigten in neuen Kontrollformen untergraben die Vorstellung einer Partnerschaft von Angestellten und Unternehmen/Management und stehen in der Praxis immer öfter im Widerspruch zur bisher hegemonialen Deutung des betrieblichen Interessengefüges als symbiotisches Verhältnis von Belegschaft und Unternehmen.

Die Veränderung der Interessenidentitäten verläuft keineswegs linear oder gar im Sinne eines Entwicklungssprungs. So enthalten die neu entstehenden Interessenidentitäten vieler Befragter weiterhin Versatzstücke ihrer beitragsorientierten Vergangenheit. Dennoch ist die grundlegende Entwicklungsrichtung eindeutig: Mehr denn je sehen sich nicht nur die qualifizierten Angestellten, sondern insbesondere auch die Hochqualifizier- ten, wenn auch auf spezifische und oft widersprüchliche Weise, auf dem Weg zu normalen Arbeitnehmern. Dies markiert einen grundlegenden Bruch in der Entwicklung der Interessenidentitäten von Angestellten.

Bei genauerer Betrachtung verläuft dieser Prozess der Neuorientierung zwischen zwei Polen - auf der einen Seite gibt es Beschäftigte, die wir „Arbeitnehmer wider Willen" nennen, und auf der anderen Seite „manifeste Arbeitnehmer“ (Boes/Kämpf 2008, 2010). Diese beiden Strömungen sind weniger im Sinne eindeutiger Klassifizierungen zu verstehen, in die man die Beschäftigten - wie in Schubladen - einsortieren könnte. Vielmehr sind sie als zwei gegensätzliche Pole zu begreifen, welche die Richtung und Dynamik der Neuorientierungsprozesse strukturieren. Beide Typen beschreiben Beschäftigte, die vormals beitragsorientiert waren und erst in der Auseinandersetzung mit neuen Lohnarbeitserfahrungen ihre Interessenidentität in Richtung neuer Arbeitnehmertypen verändern.

Die „Arbeitnehmer wider Willen“ erleben ihr neues Arbeitnehmersein vor allem als einen Verlust ihrer bisherigen Sonderstellung, dem sie weitgehend machtlos gegenüberstehen. Die Erkenntnis, im Sinne eines kollektiven Abstiegs nun offensichtlich doch „nur“ Arbeitnehmer zu sein, wird dabei zum Eingeständnis der eigenen Ohnmacht. Der für diese Strömung charakteristische romantische Blick zurück blockiert in der Folge neue Handlungsstrategien und führt $\mathrm{zu}$ Konformität und Anpassung. Ohnmacht und der Verlust von Handlungsfähigkeit kennzeichnen die „Arbeitnehmer wider Willen" und konstituieren den Kern ihres Arbeitnehmerseins. Zahlenmäßig bilden sie die Mehrheit. Dem gegenüber stehen die „manifesten Arbeitnehmer“ (siehe dazu bereits Boes/Trinks 2006). Der Begriff des „manifesten Arbeitnehmers“ beschreibt Beschäftigte, die die neue Identität, Arbeitnehmer zu sein, positiv anerkennen und zum Ausgangspunkt neuer Handlungsstrategien machen. Sie sind häufig „Überzeugungstäter“, die es sich auf Grundlage hoher individueller Primärmacht leisten können, sich offensiv und selbstbewusst zu ihrem Arbeitnehmersein zu bekennen. Ausgangspunkt für diese Neuorientierung ist insbesondere eine bewusste Reflexion von Interessengegensätzen im Unternehmen. Für die manifesten Arbeitnehmer 
kommt das Eingeständnis, Arbeitnehmer zu sein, oftmals einer inneren Befreiung gleich, die es ihnen erlaubt, neue Formen des Interessenhandelns zu erschließen.

\section{Herausforderungen für die Arbeitspolitik: Arbeitsbeziehungen am Scheideweg}

Mit Blick auf die künftige Dynamik der Arbeitsbeziehungen in den Angestelltenbereichen geht die empirisch eindeutige Erosion der Beitragsorientierung somit offensichtlich mit zwei gegenläufigen Entwicklungsszenarien einher. Auf der einen Seite lässt die Verbreitung des „Arbeitnehmers wider Willen" vor allem solche Szenarien plausibel erscheinen, die dem Leitbild des „geduldigen Arbeiters“ (Kadritzke 1982) folgen und im Sinne individueller Anpassung eine Entsolidarisierung in Angestelltenbereichen beinhalten. Auf der anderen Seite ist in der Erscheinung des „manifesten Arbeitnehmers“ eine gegenläufige Entwicklungstendenz angelegt. Diese Beschäftigtengruppe ist nicht nur bereit, für die eigenen Interessen einzustehen, sondern bezieht sich positiv auf die Mitbe- stimmung und die Vorstellung kollektiver Strategien der Interessendurchsetzung.

In der Praxis besteht zwischen den beiden Strömungen keine chinesische Mauer; welche der beiden Strömungen sich in der Praxis durchsetzen wird, wird letztlich vom konkreten Verlauf der realen Auseinandersetzungen abhängen - und damit vor allem von der Frage, ob Gewerkschaften und qualifizierte wie hochqualifizierte Angestellte in eine produktive Beziehung zueinander finden werden. Zugespitzt ausgedrückt, stehen damit die Arbeitsbeziehungen im Angestelltenbereich an einem Scheideweg: Als Negativszenario zeichnet sich eine neue Ökonomie der Entwertung und der Ohnmacht der Beschäftigten ab, als Positivszenario denkbar ist jedoch auch eine neue Kultur der Solidarität in Angestelltenbereichen, die eine neue Qualität der Mitbestimmung und gestiegene Handlungsfähigkeit beinhaltet. Vor diesem Hintergrund werden Angestellte und Hochqualifizierte mehr denn je zu einer strategischen Zielgruppe von Gewerkschaften und Betriebsräten.

Um mit den Hochqualifizierten und Angestellten eine produktive Beziehung aufzubauen, ist es notwendig, dass sich Gewerkschaften auch auf den besonderen Habitus dieser Zielgruppe einstellen und lernen, „ihre Sprache zu sprechen“. Hier wurden in der Vergangenheit auch bereits wichtige Fortschritte erzielt. Das Bemühen um Anschlussfähigkeit sollte jedoch nicht den Blick auf eine weitere zentrale Ursache der bislang anhaltenden Distanz zu den Gewerkschaften verstellen. Folgt man unseren Analysen, beruht die mangelnde Attraktivität kollektiver Strategien des Interessenhandelns vor allem darauf, dass Gewerkschaften angesichts der Globalisierung selbst als machtlose und handlungsunfähige Akteure gesehen werden. Für Gewerkschaften und Betriebsräte bedeutet dies, dass es neben ihrem Bemühen, Anschlussfähigkeit zu erlangen, insbesondere darum gehen muss, auch in Bereichen der Angestelltenarbeit Handlungsfähigkeit zu entwickeln und zu demonstrieren. Dazu gilt es, an den Widerspruchserfahrungen der Beschäftigten im Arbeitsalltag anzuknüpfen und Auswege aus den individuellen (Handlungs-)Dilemmata anzubieten. Anders ausgedrückt: Bei der Frage nach einer Annäherung von Gewerkschaften und Angestelltengruppen geht es darum, ob es den Gewerkschaften gelingt, im „System permanenter Bewährung" kollektive Alternativen zur erlebten Ohnmacht und Unsicherheit zu formulieren - und wie man als Gewerkschaft ein authentischer und handlungsfähiger $\mathrm{Ge}$ genpol zu einer fortschreitenden Ökonomie der Entwertung werden kann.

\section{LITERATUR}

Abel, J./Ittermann, P./Pries, L. (2005): Erwerbsregulierung in hochqualifizierter Wissensarbeit - individuell und kollektiv, diskursiv und partizipativ, in: Industrielle Beziehungen 1, S. 28-50

Ahlers, E./Öz, F./Ziegler, A. (2007): Standortverlagerung in Deutschland: einige empirische und politische Befunde, Düsseldorf

Baethge, M./Denkinger, J./Kadritzke, U. (1995): Das FührungskräfteDilemma - Manager und industrielle Experten zwischen Unternehmen und Lebenswelt, Frankfurt am Main, New York

Baethge, M./Oberbeck, H. (1986): Zukunft der Angestellten - Neue Technologien und berufliche Perspektiven in Büro und Verwaltung, Frankfurt am Main/New York

Baukrowitz, A./Boes, A. (1996): Arbeit in der "Informationsgesellschaft“ - Einige grundsätzliche Überlegungen aus einer (fast schon) ungewohnten Perspektive, in: Schmiede, R. (Hrsg.): Virtuelle Arbeitswelten - Arbeit, Produktion und Subjekt in der "Informationsgesellschaft“", Berlin, S. 129-158
Beck, U. (2008): Jenseits von Klasse und Nation. Individualisierung und Transnationalisierung sozialer Ungleichheiten, in: Soziale Welt 4, S. 301-326 Berger, U./Offe, C. (1981): Das Rationalisierungsdilemma der Angestelltenarbeit - Arbeitssoziologische Überlegungen zur Erklärung des Status von kaufmännischen Angestellten aus der Eigenschaft ihrer Arbeit als "Dienstleistungsarbeit", in: Kocka, J. (Hrsg.): Angestellte im europäischen Vergleich, Göttingen, S. 39-58

Bleicher, S. (1985): Probleme und Perspektiven gewerkschaftlicher Angestelltenpolitik, in: Gewerkschaftliche Monatshefte 10, S. 577-583 Boes, A. (1996): Formierung und Emanzipation - Zur Dialektik der Arbeit in der "Informationsgesellschaft", in: Schmiede, R. (Hrsg.): Virtuelle Arbeitswelten. Arbeit, Produktion und Subjekt in der "Informationsgesellschaft“", Berlin, S. 159-178

Boes, A. (2004): Offshoring in der IT-Industrie - Strategien der Internationalisierung und Auslagerung im Bereich Software und IT-Dienstleistungen, in: Boes, A./Schwemmle, M. (Hrsg.): Herausforderung Offshoring - Internationalisierung und Auslagerung von IT-Dienstleistungen, Düsseldorf, S. 9-140 
Boes, A. (2005): Auf dem Weg in die Sackgasse? - Internationalisierung im Feld Software und IT-Services, in: Boes, A./Schwemmle, M. (Hrsg.): Bangalore statt Böblingen? Offshoring und Internationalisierung im IT-Sektor, Hamburg, S. 13-65

Boes, A./Baukrowitz, A. (2002): Arbeitsbeziehungen in der ITIndustrie - Erosion oder Innovation der Mitbestimmung?, Berlin Boes, A./Bultemeier, A. (2008): Informatisierung - Unsicherheit Kontrolle, in: Dröge, K./Marrs, K./Menz, W. (Hrsg.): Die Rückkehr der Leistungsfrage. Leistung in Arbeit, Unternehmen und Gesellschaft, Berlin, S. 59-91

Boes, A./Kämpf, T. (2008): Hochqualifizierte in einer globalisierten Arbeitswelt - Von der Erosion der "Beitragsorientierung " zu einer neuen Arbeitnehmeridentität, in: Arbeits- und Industriesoziologische Studien 2, S. 44-67

Boes, A./Kämpf, T. (2010): Offshoring und eine neue Phase der Internationalisierung von Arbeit. Endbericht für die Hans-Böckler-Stiftung (im Erscheinen)

Boes, A./Kämpf, T./Marrs, K./Trinks, K. (2007): "The World is flat" - Nachhaltige Internationalisierung als Antwort auf die Herausforderungen einer globalen Dienstleistungswirtschaft, Arbeitspapier 3 des Projekts Export IT (ISF München), München

Boes, A./Kämpf, T./Marrs, K./Trinks, K. (2008): Der IT-Standort Deutschland und die Chancen einer nachhaltigen Internationalisierung, Arbeitspapier 4 des Projekts Export IT (ISF München), München Boes, A./Trinks, K. (2006): Theoretisch bin ich frei! Interessenhandeln und Mitbestimmung in der IT-Industrie, Berlin

Braverman, H. (1977): Die Arbeit im modernen Produktionsprozeß, Frankfurt am Main/New York

Castells, M. (1996): The Rise of the Network Society, Cambridge

Crozier, M./Friedberg, E. (1979): Macht und Organisation - Die Zwänge kollektiven Handelns, Königstein

Dahrendorf, R. (2009): Die Derivatisierung der Welt und ihre Folgen. Ein Gespräch mit Ralf Dahrendorf zum 80. Geburtstag, in: Leviathan 2, S. $177-186$

Ernst, D. (2008): A new Geography of Knowledge? Asia's role in global innovation networks, Policy Studies 54, Honolulu

Faust, M./Jauch, P./Notz, P. (2000): Befreit und entwurzelt: Führungskräfte auf dem Weg zum „internen Unternehmer" ${ }^{\prime}$ München/Mering Flecker, J./Huws, U. (Hrsg.) (2004): Asian Emergence - The World's Back Office?, IES Report 409, Brighton

Friedman, A. (1977): Responsible Autonomy versus Direct Control over the Labour Process, in: Capital and Class 1, S. 43-57

Hartmann, M. (2009): Die transnationale Klasse. Mythos oder Realität?, in: Soziale Welt 3, S. 286-305

Heidenreich, M./Töpsch, K. (1998): Die Organisation der Arbeit in der Wissensgesellschaft, in: Industrielle Beziehungen 1, S. 13-44

Hoose, F./Jeworutzki, S./Pries, L. (2009): Führungskräfte und betriebliche Mitbestimmung. Zur Praxis der Partizipation am Beispiel der chemischen Industrie, Frankfurt am Main/New York
Hürtgen, S./Lüthje, B./Schumm, W./Sproll, M. (2009): Von Silicon Valley nach Shenzhen. Globale Produktion und Arbeit in der IT-Industrie, Hamburg

Huws, U./Dahlmann, S. (2009): Global Restructuring of Value Chains and Class Issues, in: Interventions Économiques 39, S. 1-27

Kadritzke, U. (1982): Angestellte als Lohnarbeiter. Kritischer Nachruf auf die deutsche Kragenlinie, in: Schmidt, G./Braczyk, H.-J./Knese-

beck, J. von (Hrsg.): Sonderheft 24 der Kölner Zeitschrift für Soziologie und Sozialpsychologie, S. 219-249

Kämpf, T. (2008): Die neue Unsicherheit - Die Folgen der Globalisierung für hochqualifizierte Arbeitnehmer, Frankfurt am Main Kotthoff, H. (1997): Führungskräfte im Wandel der Firmenkultur: Quasi-Unternehmer oder Arbeitnehmer?, Berlin

Kotthoff, H./Wagner, A. (2008): Die Leistungsträger. Führungskräfte im Wandel der Firmenkultur - eine Follow-up-Studie, Berlin

Kratzer, N./Nies, S. (2009): Neue Leistungspolitik bei Angestellten. ERA, Leistungssteuerung, Leistungsentgelt, Berlin

Lange, H./Städler, A./Feseker, K. (2005): Intern@work - Interessenregulierung in der New Economy, Bremen

Littek, W./Heisig, U. (1995): Taylorism never got hold of skilled white-collar work in Germany, in: Littek, W./Charles, T. (Hrsg.): The new division of labour: emerging forms of work organisation in international perspective, Berlin, S. 373-395

Lüthje, B. (2006): Wintelismus zum „China-Preis". Wohin treibt das Produktionsmodell der IT-Industrie?, in: Baukrowitz, A./Berker, T./ Boes, A./Pfeiffer, S./Schmiede, R./Will, M. (Hrsg.): Informatisierung der Arbeit - Gesellschaft im Umbruch, Berlin, S. 346-357

Martens, H. (2006): Nach dem Ende des Hype. Zwischen Interessenvertretungsarbeit und Arbeitspolitik, Münster

Martens, H./Dechmann, U. (2010): Am Ende der Deutschland AG. Standortkonflikte im Kontext einer neuen Politik der Arbeit, Münster Mehlis, P. (2008): „Vom kreativen Chaos zur effizienten Organisation". Gestaltung und Regulierung hochqualifizierter Arbeit in IT- und Biotechnologieunternehmen, München/Mering

Minssen, H. (2009): Bindung und Entgrenzung. Eine Soziologie international tätiger Manager, München/Mering

Raeder, S./Grote, G. (2001): Flexibilität ersetzt Kontinuität. Veränderte psychologische Kontrakte und neue Formen persönlicher Identität, in: Arbeit 3, S. 352-364

Rousseau, D. (1995): Psychological contracts in organizations. Understanding written and unwritten agreements, London

Urban, H.-J. (1989): Angestellte und gewerkschaftliche Gegenmacht. Zur Angestelltenpolitik der IG Metall in der Umbruchkrise, Marburg Vester, M./Teiwes-Kügler, C./Lange-Vester, A. (2007): Die neuen Arbeitnehmer. Zunehmende Kompetenzen - wachsende Unsicherheit, Hamburg

Voß, G./Pongratz, H. J. (1998): Der Arbeitskraftunternehmer Eine neue Grundform der "Ware Arbeitskraft"?, in: Kölner Zeitschrift für Soziologie und Sozialpsychologie 1, S. 131-158 